\title{
Formulation and Evaluation of Extended Release Matrix Tablets of Tenatoprazole Sodium using Synthetic Polymers
}

\author{
B. Divya ${ }^{1 *}$, J. Sreekanth ${ }^{2}$, D. Satyavati ${ }^{1}$ \\ 'Brilliant Group of Institutions (Integrated Campus), Abdullapurmet, Hayathnagar, R.R Dist, Telangana, India. \\ ${ }^{2}$ Progenerics Pharma Pvt. Ltd., Hyderabad, Telangana, India.
}

\begin{abstract}
Extended release formulations are designed to release their medication in a controlled manner at a predetermined rate, duration and location to achieve and maintain optimum therapeutic blood levels of a drug. The present investigation is aimed to formulate the extended release matrix tablets of Tenatoprazole sodium with various grades of different polymers like Carbopol, HPMC and Eudragit grades. The tablets were prepared by wet granulation technique. The prepared ER Matrix tablets were evaluated for various physico chemical parameters. All the formulations resulted in acceptable Pharmacopoeia limits. In-vitro drug release studies (USP dissolution rate test apparatus $11,75 \mathrm{rpm}, 37^{\circ} \mathrm{C} \pm 0.5^{\circ} \mathrm{C}$ ) using $0.1 \mathrm{~N}$ hydrochloric acid (1.2 PH) for first 2 hrs and phosphate buffer (PH 6.8) as a dissolution medium $(900 \mathrm{ml})$ for the next $12 \mathrm{hrs}$. Among all the formulation F-5/drug:
\end{abstract}

Carbopol-974P-NF in ratio of 1:1.5) shows better result upto 12 hours of the drug release was found to be $99.47 \pm 0.22$ so it's an Optimized formulation. Key words: Carbopol, ER Matrix, Eudragit, Methocel, Tenatoprazole sodium, Wet granulation.

Correspondence

B. Divya*,

Brilliant Group of Institutions (Integrated Campus), Abdullapurmet, Hayathnagar, R.R Dist, Telangana, India.

Phone: +919885822399

Email: divya.balne444@gmail.com

DOI: 10.5530/jyp.2020.12s.44

\section{INTRODUCTION}

Proton pump inhibitors (PPIs) are very effective in the treatment of symptoms and healing of erosive and ulcerative disease in the spectrum of acid-related disorders. PPIs produce significantly more effective and prolonged acid suppression than $\mathrm{H} 2$-receptor antagonists (H2-RAs) and currently available PPIs, at higher daily doses or administration frequencies, can maintain a $\mathrm{pH}>4$ for up to $16-18 \mathrm{~h} /$ day. It has been shown that healing in acid-related disorders is directly related to the degree and duration of acid suppression and the length of treatment. However, a significant number of patients with acid-related disorders do not adequately respond to once or even twice daily PPI therapy. ${ }^{1} \mathrm{~A}$ suboptimal symptomatic response to PPI therapy commonly leads the physician to double the PPI dose. The pharmacokinetic properties of the currently available PPIs limit their ability to produce prolonged acid suppression, leading to markedly reduced efficacy during the nocturnal period (Figure 1 and 2). ${ }^{2}$ PPIs produce greater acid suppression if taken before food, because the maximum number of acid pumps is inserted into the canalicular membrane and they require an acidic intra-canalicular environment with active acid secretion in the gastric parietal cell to permit conversion to the active sulphenamide and covalent binding of the sulphenamide to effect inhibition of acid secretion. As current PPIs all have similar plasma half-lives between 1 and $2 \mathrm{~h}$, any proton pumps synthesized or activated after the plasma level of the PPI falls below threshold will not be blocked from secreting acid, with the possible exception of sulphenamide derivatives, trapped at high concentration in the secretory canaliculi. ${ }^{3}$

Tenatoprazole is a new PPI that has a prolonged plasma half-life of around $9 \mathrm{~h}$ which is 5-7 fold longer than the currently available PPIs. In contrast to current PPIs, characterized by pyridine and benzimidazole moieties, tenatoprazole has an imidazopyridine ring in place of the benzimidazole moiety. The results of several studies indicate that racemic tenatoprazole produces more prolonged suppression of intragastric acidity than esomeprazole, an S-enantiomer of omeprazole that is more effective than its parent racemic compound. STU-Na is the S-enantiomer of tenatoprazole and, preliminary pharmacological studies indicate that it too may be more effective than its parent racemic compound. ${ }^{4,5}$

\section{MATERIALS AND METHODS}

Materials: Tenatoprazole sodium was received as a gift sample from LARA DRUGS PVT LTD, Hyderabad. Carbopol-941P, Carbopol-974P-NF, Methocel K4M, Methocel K100 LV, Eudragit-S 100, L-100, RS100, RL-100 was supplied by Yarrow Chem Products, Mumbai. PVP-K 30, IPA, Aerosil, Magnesium Stearate and Microcrystalline Cellulose was supplied by Signet Chem Mumbai.

\section{METHODS}

\section{Preformulation Studies}

Standardization of Tenatoprazole sodium by UV-Visible Spectrophotometry

a) In $0.1 \mathrm{~N}$ Hcl Solution

i) Preparation of stock solution: Stock solution $100 \mu \mathrm{g} / \mathrm{ml}$ Tenatoprazole sodium was prepared in $0.1 \mathrm{~N} \mathrm{Hcl}$ solution. This solution was approximately diluted with $0.1 \mathrm{~N} \mathrm{Hcl}$ to obtain a concentration of $10 \mu \mathrm{g} /$ $\mathrm{ml}$. The resultant solution was scanned in range of $200-400 \mathrm{~nm}$ using UV double beam spectrophotometer (Lab India UV-3000+).

ii) Standard calibration of Tenatoprazole sodium in $0.1 \mathrm{~N} \mathrm{Hcl:} 100 \mathrm{mg}$ of Tenatoprazole sodium was accurately weighed and dissolved in $100 \mathrm{ml}$ of $0.1 \mathrm{~N} \mathrm{Hcl}$ to obtain a concentration of $1000 \mu \mathrm{g} / \mathrm{ml}$. From the above $10 \mathrm{ml}$ was withdrawn and diluted to $100 \mathrm{ml}$ to obtain a concentration of $100 \mu \mathrm{g} / \mathrm{ml}$. From this stock solution aliquots of $0.5 \mathrm{ml}, 1 \mathrm{ml}, 1.5 \mathrm{ml}, 2 \mathrm{ml}$ 
and $2.5 \mathrm{ml}$ were diluted in $10 \mathrm{ml}$ volumetric flask with phosphate buffer to give concentrations in range of $5 \mu \mathrm{g} / \mathrm{ml}$ to $25 \mu \mathrm{g} / \mathrm{ml}$ respectively, absorbance was measured at $236 \mathrm{~nm}$.

\section{b) In pH 6.8 phosphate Buffer (Table 4)}

i) Preparation of stock solution: Stock solution $100 \mu \mathrm{g} / \mathrm{ml}$ of Tenatoprazole sodium was prepared in phosphate buffer of $\mathrm{pH}$ 6.8. This solution was

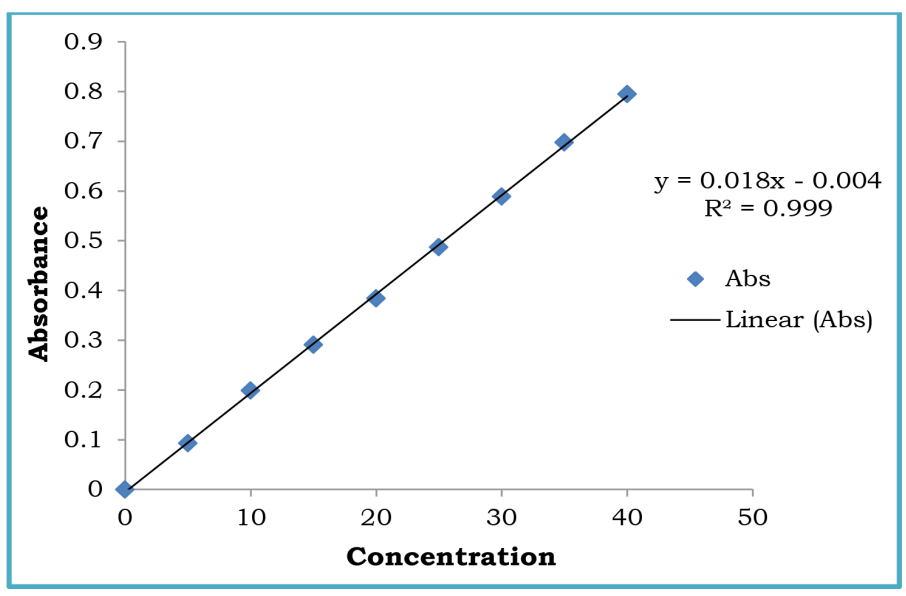

Figure 1: Standard graph of Tenatoprazole sodium pH 6.8 phosphate buffer.

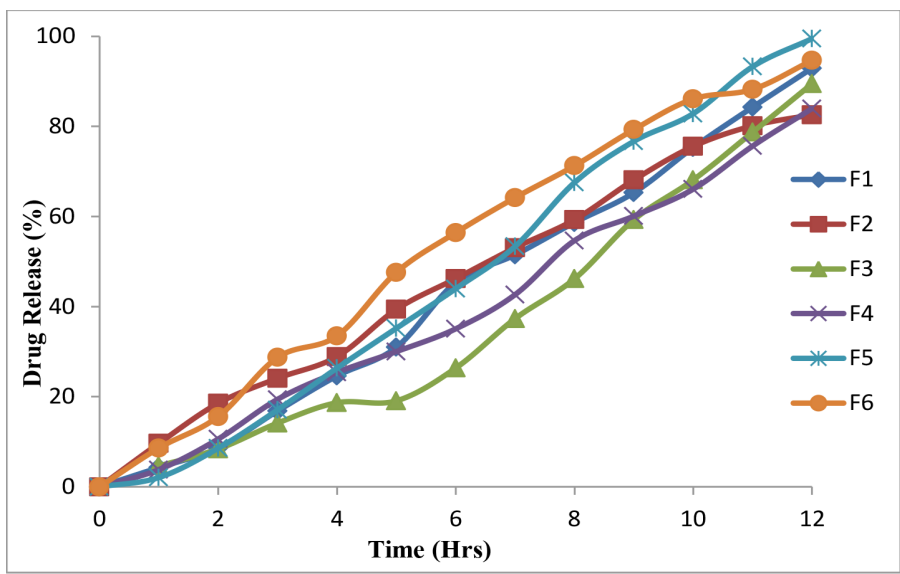

Figure 2: Dissolution profiles of Formulations F1-F6. approximately diluted with phosphate buffer of $\mathrm{pH} 6.8$ to obtain a concentration of $10 \mu \mathrm{g} / \mathrm{ml}$. The resultant solution was scanned in range of 200-400nm using UV double beam spectrophotometer (Lab India UV-3000+).

ii) Standard calibration of Tenatoprazole sodium in phosphate buffer of pH 6.8: $100 \mathrm{mg}$ of Tenatoprazole sodium was accurately weighed and dissolved in $100 \mathrm{ml}$ of $\mathrm{pH} 6.8$ phosphate buffer to obtain a concentration of $1000 \mu \mathrm{g} / \mathrm{ml}$. From the above $10 \mathrm{ml}$ was withdrawn and diluted to $100 \mathrm{ml}$ to obtain a concentration of $100 \mu \mathrm{g} / \mathrm{ml}$. From this stock solution aliquots of $0.5 \mathrm{ml}, 1 \mathrm{ml}, 1.5 \mathrm{ml}, 2 \mathrm{ml}$ and $2.5 \mathrm{ml}$ were diluted in $10 \mathrm{ml}$ volumetric flask with phosphate buffer to give concentrations in range of $5 \mu \mathrm{g} / \mathrm{ml}$ to $25 \mu \mathrm{g} / \mathrm{ml}$ respectively, absorbance was measured at $238 \mathrm{~nm} .^{6}$

\section{FORMULATION DEVELOPMENT OF TABLETS}

\section{Preparation of Tablets}

Wet granulation method: All the powders were passed through 80 mesh. Required quantities of Tenatoprazole sodium, polymer, binder (PVPK30), Sodium bicarbonate were mixed thoroughly and a sufficient volume of granulating agent (Isopropyl alcohol) was added slowly. After enough cohesiveness was obtained, the mass was sieved through 22/44 mesh. The granules were dried at $40^{\circ} \mathrm{C}$ for $12 \mathrm{hrs}$. Once, dry the granules retained on 44 mesh were mixed with $10 \%$ of fine granules that passed through 44 mesh (Figure 3,4). Aerosil and magnesium stearate were added as glidant and lubricant. Finally MCC is added to make up $250 \mathrm{mg}$. In all formulations, the amount of the active ingredient is equivalent to $40 \mathrm{mg}$ of Tenatoprazole sodium (Table 1,2)

\section{Evaluation of Tenatoprazole sodium ER matrix tablets Evaluation of granules}

\section{Angle of repose}

The angle of repose of granules was determined by the funnel method. The granules were allowed to flow through the funnel freely onto the surface. The diameter of the powder cone was measured and angle of repose was calculated using the following equation.

$$
\operatorname{Tan} \theta=\mathrm{h} / \mathrm{r}
$$

Where $\mathrm{h}$ and $\mathrm{r}$ are the height and radius of the powder cone.

\section{Bulk Density}

Both loose bulk density and tapped bulk density were determined and calculated by using the following formulas.

Table 1: Composition of extended release matrix tablets of Tenatoprazole sodium using synthetic polymers.

\begin{tabular}{|c|c|c|c|c|c|c|c|c|c|}
\hline INGREDIENTS & F1 & F2 & F3 & F4 & F5 & F6 & F7 & F8 & F9 \\
\hline Tenatoprazole sodium & 40 & 40 & 40 & 40 & 40 & 40 & 40 & 40 & 40 \\
\hline Carbopol-941P & 40 & 60 & 80 & --- & --- & --- & --- & --- & --- \\
\hline Carbopol-974P-NF & --- & --- & --- & 40 & 60 & 80 & --- & --- & --- \\
\hline Methocel K4M & --- & --- & --- & --- & --- & --- & 40 & 60 & --- \\
\hline Methocel K100LV & --- & --- & --- & --- & --- & --- & --- & --- & 40 \\
\hline PVP-K 30 & 12.5 & 12.5 & 12.5 & 12.5 & 12.5 & 12.5 & 12.5 & 12.5 & 12.5 \\
\hline Sodium bicarbonate & 10 & 10 & 10 & 10 & 10 & 10 & 10 & 10 & 10 \\
\hline IPA & QS & QS & QS & QS & QS & QS & QS & QS & QS \\
\hline Aerosil & 5 & 5 & 5 & 5 & 5 & 5 & 5 & 5 & 5 \\
\hline Mg.Stearate & 5 & 5 & 5 & 5 & 5 & 5 & 5 & 5 & 5 \\
\hline MCC & 137.5 & 117.5 & 97.5 & 137.5 & 117.5 & 97.5 & 137.5 & 117.5 & 137.5 \\
\hline Total Weight(mg) & 250 & 250 & 250 & 250 & 250 & 250 & 250 & 250 & 250 \\
\hline
\end{tabular}


Table 2: Composition of extended release matrix tablets of Tenatoprazole sodium using synthetic polymers.

\begin{tabular}{|c|c|c|c|c|c|c|c|c|c|}
\hline INGREDIENTS & F10 & F11 & F12 & $\mathrm{F} 13$ & F14 & F15 & F16 & F17 & F18 \\
\hline Tenatoprazole sodium & 40 & 40 & 40 & 40 & 40 & 40 & 40 & 40 & 40 \\
\hline Eudragit-S 100 & --- & 40 & 60 & --- & --- & --- & --- & --- & --- \\
\hline Eudragit-L 100 & --- & --- & --- & 40 & 60 & --- & ---- & --- & --- \\
\hline Eudragit-RS 100 & --- & --- & --- & & & 40 & 60 & --- & --- \\
\hline Eudragit-RL 100 & 60 & --- & --- & --- & --- & --- & --- & 40 & 60 \\
\hline Sodium bicarbonate & 10 & 10 & 10 & 10 & 10 & 10 & 10 & 10 & 10 \\
\hline PVP-K 30 & 12.5 & 12.5 & 12.5 & 12.5 & 12.5 & 12.5 & 12.5 & 12.5 & 12.5 \\
\hline IPA & QS & QS & QS & QS & QS & QS & QS & QS & QS \\
\hline Aerosil & 5 & 5 & 5 & 5 & 5 & 5 & 5 & 5 & 5 \\
\hline Mg.Stearate & 5 & 5 & 5 & 5 & 5 & 5 & 5 & 5 & 5 \\
\hline MCC & 117.5 & 137.5 & 117.5 & 137.5 & 117.5 & 137.5 & 117.5 & 137.5 & 117.5 \\
\hline Total Weight(mg) & 250 & 250 & 250 & 250 & 250 & 250 & 250 & 250 & 250 \\
\hline
\end{tabular}

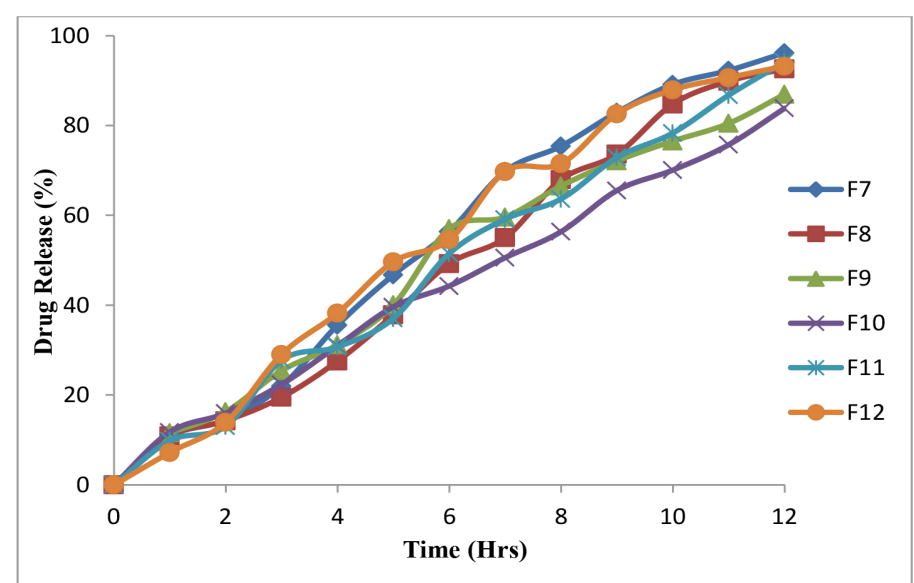

Figure 3: Dissolution profiles of Formulations F7-F12.

Table 3: Observations for graph of Tenatoprazole sodium in $0.1 \mathrm{~N} \mathrm{HCl}$ (236nm).

\begin{tabular}{ccc}
\hline S.No & Concentration $(\mu \mathrm{g} / \mathrm{ml})$ & Absorbance $(\mathrm{nm})$ \\
\hline 1 & 0 & 0 \\
2 & 5 & 0.111 \\
3 & 10 & 0.216 \\
4 & 15 & 0.303 \\
5 & 20 & 0.406 \\
6 & 25 & 0.511 \\
\hline
\end{tabular}

Table 4: Observations for graph of Tenatoprazole sodium in pH 6.8 phosphate buffer (238nm).

\begin{tabular}{ccc} 
S.No & Concentration $(\mu \mathrm{g} / \mathrm{ml})$ & Absorbance $(\mathrm{nm})$ \\
\hline 1 & 0 & 0 \\
2 & 5 & 0.092 \\
3 & 10 & 0.200 \\
4 & 15 & 0.289 \\
5 & 20 & 0.385 \\
6 & 25 & 0.486 \\
\hline
\end{tabular}

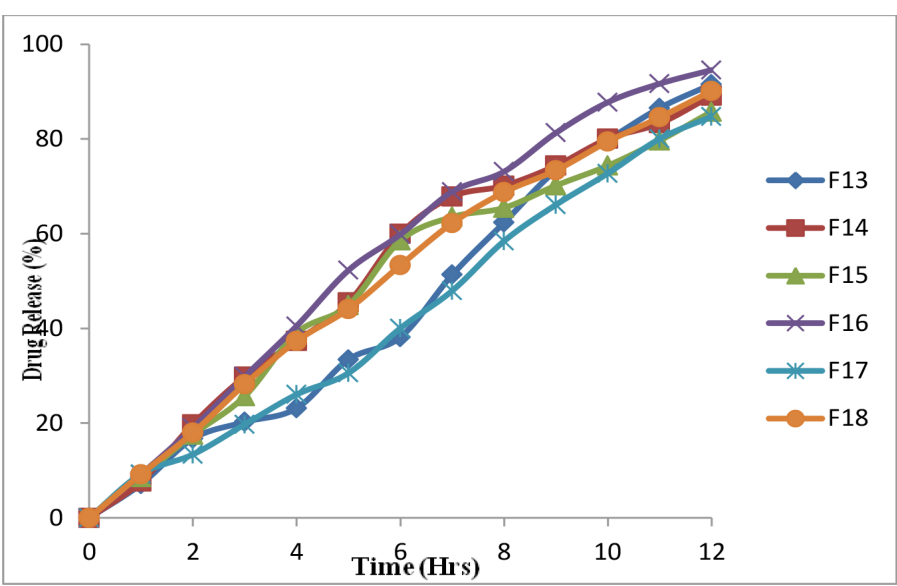

Figure 4: Dissolution profiles of Formulations F13-F18.

$\mathrm{LBD}=$ weight of the powder / volume of the packing

$\mathrm{TBD}=$ weight of the powder / tapped volume of the packing

Compressibility Index: The compressibility index of the granules was determined by Carr's compressibility index.

$$
\text { Carr's index }(\%)=[\text { TBD-LBD }] \times 100 / \text { TBD }
$$

Hausner's ratio: The Hausner's ratio is a number that is correlated to the flowability of a powder or granular material. The ratio of tapped density to bulk density of the powders is called the Hausner's ratio. It is calculated by the following equation. ${ }^{\text {? }}$

$$
\mathrm{H}=\text { Tapped density / Bulk density }
$$

\section{Evaluation of tablets}

Average weight: To study weight variation, 20 tablets of each formulation were weighed using an electronic balance (Sartorius India, limited) and the test was carried according to the Indian Pharmacopoeia. ${ }^{8}$

Drug Content: Five tablets were weighed individually and the drug was extracted in $\mathrm{pH} 6.8$ phosphate buffer. The drug content was determined according to the IP.

Hardness and Friability: The hardness and friability were determined using the Monsanto hardness tester (Cadmach, Ahmedabad, India) and the friability testing apparatus (Indian equipments, Mumbai, India), respectively. ${ }^{9}$ 
Table 5: PRE Compression Parameters.

\begin{tabular}{|c|c|c|c|c|c|}
\hline بْ & 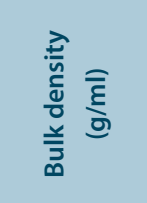 & 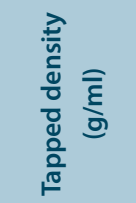 & 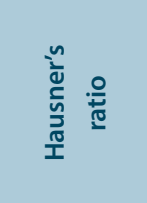 & 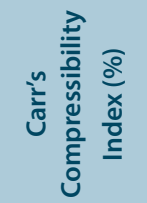 & 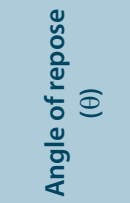 \\
\hline F1 & $0.426 \pm 0.28$ & $0.524 \pm 0.39$ & $1.230 \pm 0.32$ & $18.70 \pm 0.37$ & $24.12 \pm 0.24$ \\
\hline $\mathrm{F} 2$ & $0.431 \pm 0.36$ & $0.537 \pm 0.17$ & $1.245 \pm 0.09$ & $23.20 \pm 0.13$ & $24.65 \pm 0.18$ \\
\hline F3 & $0.412 \pm 0.09$ & $0.479 \pm 0.15$ & $1.162 \pm 0.17$ & $13.98 \pm 0.05$ & $25.77 \pm 0.27$ \\
\hline $\mathrm{F} 4$ & $0.467 \pm 0.37$ & $0.558 \pm 0.14$ & $1.371 \pm 0.16$ & $16.51 \pm 0.68$ & $26.09 \pm 0.34$ \\
\hline F5 & $0.574 \pm 0.33$ & $0.602 \pm 0.16$ & $1.048 \pm 0.58$ & $12.98 \pm 0.36$ & $27.18 \pm 0.06$ \\
\hline F6 & $0.471 \pm 0.15$ & $0.588 \pm 0.17$ & $1.248 \pm 0.05$ & $19.89 \pm 0.24$ & $25.08 \pm 0.17$ \\
\hline F7 & $0.543 \pm 0.28$ & $0.646 \pm 0.15$ & $1.189 \pm 0.11$ & $15.94 \pm 0.47$ & $23.33 \pm 0.26$ \\
\hline F8 & $0.481 \pm 0.32$ & $0.542 \pm 0.16$ & $1.126 \pm 0.08$ & $19.06 \pm 0.14$ & $25.11 \pm 0.25$ \\
\hline F9 & $0.452 \pm 0.11$ & $0.629 \pm 0.23$ & $1.391 \pm 0.23$ & $21.67 \pm 0.03$ & $24.72 \pm 0.43$ \\
\hline F10 & $0.536 \pm 0.34$ & $0.650 \pm 0.26$ & $1.212 \pm 0.17$ & $17.46 \pm 0.48$ & $26.67 \pm 0.28$ \\
\hline F11 & $0.564 \pm 0.22$ & $0.602 \pm 0.19$ & $1.067 \pm 0.14$ & $20.59 \pm 0.43$ & $26.13 \pm 0.43$ \\
\hline F12 & $0.581 \pm 0.37$ & $0.599 \pm 0.15$ & $1.031 \pm 0.08$ & $17.29 \pm 0.28$ & $24.52 \pm 0.44$ \\
\hline F13 & $0.431 \pm 0.07$ & $0.497 \pm 0.17$ & $1.153 \pm 0.17$ & $19.83 \pm 0.16$ & $22.06 \pm 0.13$ \\
\hline F14 & $0.544 \pm 0.19$ & $0.602 \pm 0.04$ & $1.106 \pm 0.99$ & $21.05 \pm 0.25$ & $26.64 \pm 0.08$ \\
\hline F15 & $0.490 \pm 0.19$ & $0.552 \pm 0.27$ & $1.126 \pm 0.12$ & $18.49 \pm 0.06$ & $25.89 \pm 0.15$ \\
\hline F16 & $0.436 \pm 0.18$ & $0.586 \pm 0.22$ & $1.344 \pm 0.16$ & $17.14 \pm 0.06$ & $27.13 \pm 0.17$ \\
\hline F17 & $0.412 \pm 0.07$ & $0.477 \pm 0.13$ & $1.157 \pm 0.08$ & $20.14 \pm 0.03$ & $22.27 \pm 0.18$ \\
\hline F18 & $0.503 \pm 0.13$ & $0.598 \pm 0.29$ & $1.1899 \pm 0.04$ & $19.61 \pm 0.11$ & $23.43 \pm 0.05$ \\
\hline
\end{tabular}

The data are presented as mean value \pm S.D. $(n=3)$

Table 6: Evaluation of sustained release matrix tablet.

\begin{tabular}{cccccc}
\hline F.Code & $\begin{array}{c}\text { Weight } \\
\text { variation }(\mathrm{mg})\end{array}$ & $\begin{array}{c}\text { Thickness } \\
(\mathrm{mm}) ¥\end{array}$ & $\begin{array}{c}\text { Hardness } \\
\left(\mathrm{kg} / \mathrm{cm}^{2}\right)\end{array}$ & $\begin{array}{c}\text { Friability } \\
(\%) \Psi\end{array}$ & $\begin{array}{c}\% \text { Drug } \\
\text { Content } €\end{array}$ \\
\hline F1 & $250 \pm 0.41$ & $3.66 \pm 0.23$ & $4.98 \pm 0.56$ & $0.78 \pm 0.04$ & $98.19 \pm 0.53$ \\
F2 & $249 \pm 0.72$ & $3.99 \pm 0.19$ & $4.79 \pm 1.18$ & $0.65 \pm 0.03$ & $99.51 \pm 0.98$ \\
F3 & $250 \pm 0.98$ & $4.13 \pm 1.44$ & $5.04 \pm 0.35$ & $0.52 \pm 0.02$ & $97.14 \pm 0.79$ \\
F4 & $248 \pm 1.16$ & $3.57 \pm 0.19$ & $4.35 \pm 0.42$ & $0.56 \pm 0.27$ & $100.48 \pm 0.07$ \\
F5 & $250 \pm 0.09$ & $3.78 \pm 0.36$ & $4.69 \pm 0.29$ & $0.57 \pm 0.25$ & $99.98 \pm 0.23$ \\
F6 & $250 \pm 1.79$ & $3.89 \pm 0.14$ & $5.14 \pm 0.35$ & $0.55 \pm 0.04$ & $97.54 \pm 1.16$ \\
F7 & $248 \pm 2.04$ & $4.07 \pm 0.17$ & $4.38 \pm 0.05$ & $0.77 \pm 0.18$ & $96.74 \pm 1.72$ \\
F8 & $250 \pm 0.21$ & $3.69 \pm 0.24$ & $4.35 \pm 0.18$ & $0.55 \pm 0.19$ & $98.14 \pm 0.98$ \\
F9 & $249 \pm 1.17$ & $4.15 \pm 1.13$ & $5.06 \pm 0.07$ & $0.43 \pm 0.11$ & $98.88 \pm 0.59$ \\
F10 & $250 \pm 0.28$ & $3.06 \pm 0.99$ & $4.58 \pm 0.16$ & $0.89 \pm 0.07$ & $101.26 \pm 0.15$ \\
F11 & $249 \pm 1.26$ & $3.67 \pm 0.34$ & $4.44 \pm 0.62$ & $0.52 \pm 0.13$ & $99.14 \pm 0.09$ \\
F12 & $250 \pm 0.89$ & $3.15 \pm 0.18$ & $4.47 \pm 0.31$ & $0.68 \pm 0.24$ & $99.22 \pm 0.08$ \\
F13 & $249 \pm 1.31$ & $3.98 \pm 0.44$ & $4.99 \pm 0.15$ & $0.67 \pm 0.05$ & $98.69 \pm 0.22$ \\
F14 & $248 \pm 1.87$ & $3.33 \pm 0.27$ & $5.11 \pm 0.13$ & $0.44 \pm 0.22$ & $101.04 \pm 0.98$ \\
F15 & $250 \pm 0.26$ & $3.59 \pm 0.63$ & $4.15 \pm 0.94$ & $0.54 \pm 0.03$ & $98.96 \pm 0.66$ \\
F16 & $250 \pm 0.94$ & $3.99 \pm 0.74$ & $4.14 \pm 1.26$ & $0.57 \pm 0.12$ & $99.16 \pm 0.03$ \\
F17 & $249 \pm 1.44$ & $3.89 \pm 0.19$ & $4.55 \pm 0.27$ & $0.75 \pm 0.54$ & $99.25 \pm 0.95$ \\
F18 & $250 \pm 0.88$ & $3.44 \pm 0.06$ & $4.96 \pm 0.24$ & $0.57 \pm 0.16$ & $98.86 \pm 0.24$ \\
\hline
\end{tabular}

$*=\mathrm{n}=20, ¥=\mathrm{n}=10, \ddot{\mathbb{K}}=\mathrm{n}=5, \psi=\mathrm{n}=10, €=\mathrm{n}=5$

\section{In-vitro release studies}

Dissolution Conditions

1) Dissolution Medium and time

a) Gastric Resistance: $0.1 \mathrm{~N} \mathrm{HCl}$ for 2 hours.

b) Dissolution: phosphate buffer $\mathrm{pH} 6.8$ for $10 \mathrm{hrs}$.

2) Volume: $900 \mathrm{ml}$

3) Apparatus: Type II (paddles)

4) Rotation Speed: $75 \mathrm{rpm}$

5) Temperature: $37^{\circ} \mathrm{C}+0.5^{\circ} \mathrm{C}$

USP dissolution apparatus type II (Electrolab TDT-08L, Mumbai, India) was used to determine the in vitro release of Tenatoprazole sodium from the prepared formulations. The dissolution medium was $900 \mathrm{ml}$ of acidic buffer $0.1 \mathrm{~N} \mathrm{HCl}$ for $2 \mathrm{~h}$ and phosphate buffer ( $\mathrm{pH} \mathrm{6.8)} \mathrm{for} 12 \mathrm{hrs}$. The tablet was kept in to the basket at $37 \pm 0.5^{\circ} \mathrm{C}$ and $75 \mathrm{rpm}$. Samples $(5 \mathrm{~mL})$ were withdrawn at regular time intervals and the dissolution medium was replaced with equal volume fresh dissolution medium. The samples were measured by UV spectrophotometer at $270 \mathrm{~nm}$ against a blank. ${ }^{10,11}$

\section{RESULTS AND DISCUSSION}

\section{Pre formulation studies}

Development of calibration curve for Tenatoprazole sodium: The scanning of the drug solution in the UV range showed maximum absorbance at $236 \mathrm{~nm}$ and hence, the calibration curve was developed at this wavelength. The values are given in Table 3.

\section{Physical characteristics of blends and tablets}

The blends of different formulations were evaluated for angle of repose, Carr's compressibility index etc., the results of Angle of repose and Carr's compressibility Index (\%) ranged from 22.06-27.2 and 12.98-23.2 respectively which showed that blends from all the formulations having good flow property. The hardness and percentage friability ranged from $4.1-5.1 \mathrm{~kg} / \mathrm{cm}^{2}$ and $0.22-0.55 \%$ respectively. (Table $5-10$ )

\section{In-vitro Dissolution Studies}

Table 7: Dissolution release profiles of Formulations (F1-F5).

\begin{tabular}{ccccccc}
\hline S. No & Time & \multicolumn{5}{c}{ Cumulative \% drug release*(\%) } \\
\cline { 3 - 7 } & (hours) & F1 & F2 & F3 & F4 & F5 \\
\hline 1 & 0 & 0 & 0 & 0 & 0 & 0 \\
2 & 1 & $4.28 \pm 0.32$ & $9.64 \pm 0.33$ & $4.65 \pm 0.27$ & $3.82 \pm 0.53$ & $2.06 \pm 0.56$ \\
3 & 2 & $8.62 \pm 0.36$ & $18.56 \pm 0.46$ & $8.39 \pm 0.48$ & $10.62 \pm 0.57$ & $8.57 \pm 0.24$ \\
4 & 3 & $16.85 \pm 0.12$ & $24.09 \pm 0.27$ & $14.16 \pm 0.63$ & $19.43 \pm 0.61$ & $17.09 \pm 0.11$ \\
5 & 4 & $24.61 \pm 0.48$ & $28.91 \pm 0.28$ & $18.67 \pm 0.87$ & $25.40 \pm 0.73$ & $26.35 \pm 0.61$ \\
6 & 5 & $30.97 \pm 0.63$ & $39.39 \pm 0.81$ & $19.08 \pm 0.38$ & $30.01 \pm 0.47$ & $35.19 \pm 0.73$ \\
7 & 6 & $45.36 \pm 0.75$ & $46.21 \pm 0.53$ & $26.36 \pm 0.59$ & $35.07 \pm 0.56$ & $43.98 \pm 0.46$ \\
8 & 7 & $51.49 \pm 0.51$ & $53.08 \pm 0.32$ & $37.33 \pm 0.79$ & $42.66 \pm 0.54$ & $53.37 \pm 0.67$ \\
9 & 8 & $58.66 \pm 0.43$ & $59.29 \pm 0.47$ & $46.17 \pm 0.71$ & $54.65 \pm 0.63$ & $67.53 \pm 0.61$ \\
10 & 9 & $65.28 \pm 0.61$ & $68.11 \pm 0.63$ & $59.29 \pm 0.42$ & $60.09 \pm 0.39$ & $76.69 \pm 0.13$ \\
11 & 10 & $75.31 \pm 0.87$ & $75.56 \pm 0.69$ & $68.12 \pm 0.68$ & $66.08 \pm 0.58$ & $82.84 \pm 0.59$ \\
12 & 11 & $84.27 \pm 0.18$ & $80.13 \pm 0.57$ & $78.71 \pm 0.54$ & $75.64 \pm 0.41$ & $93.26 \pm 0.35$ \\
13 & 12 & $92.91 \pm 0.25$ & $82.58 \pm 0.46$ & $89.45 \pm 0.71$ & $83.95 \pm 0.65$ & $99.47 \pm 0.22$ \\
\hline
\end{tabular}

The data are presented as mean value \pm S.D. $(n=3)$ 
Divya, et al.: Development of Extended Release Tablets of Tenatoprazole Sodium

Table 8: Dissolution release profiles of Formulations (F6-F10).

\begin{tabular}{ccccccc}
\hline \multirow{2}{*}{$\begin{array}{c}\text { S. } \\
\text { No }\end{array}$} & Time & \multicolumn{5}{c}{ Cumulative $\%$ drug release*(\%) } \\
\cline { 3 - 7 } & & F6 & F7 & F8 & F9 & F10 \\
\hline 1 & 0 & 0 & 0 & 0 & 0 & 0 \\
2 & 1 & $8.62 \pm 0.47$ & $10.81 \pm 0.35$ & $10.88 \pm 0.58$ & $11.60 \pm 0.32$ & $11.78 \pm 0.47$ \\
3 & 2 & $15.60 \pm 0.16$ & $14.44 \pm 0.41$ & $14.29 \pm 0.69$ & $16.29 \pm 0.47$ & $15.96 \pm 0.59$ \\
4 & 3 & $28.73 \pm 0.49$ & $21.99 \pm 0.36$ & $19.51 \pm 0.17$ & $25.45 \pm 0.36$ & $22.38 \pm 0.52$ \\
5 & 4 & $33.49 \pm 0.41$ & $35.58 \pm 0.59$ & $27.57 \pm 0.43$ & $31.21 \pm 0.45$ & $30.97 \pm 0.48$ \\
6 & 5 & $47.61 \pm 0.54$ & $46.76 \pm 0.33$ & $37.85 \pm 0.65$ & $40.07 \pm 0.48$ & $39.66 \pm 0.36$ \\
7 & 6 & $56.41 \pm 0.45$ & $56.37 \pm 0.64$ & $49.26 \pm 0.77$ & $57.02 \pm 0.53$ & $44.28 \pm 0.55$ \\
8 & 7 & $64.18 \pm 0.68$ & $69.83 \pm 0.38$ & $55.03 \pm 0.65$ & $59.63 \pm 0.45$ & $50.58 \pm 0.64$ \\
9 & 8 & $71.26 \pm 0.61$ & $75.42 \pm 0.52$ & $67.95 \pm 0.52$ & $66.55 \pm 0.67$ & $56.37 \pm 0.13$ \\
10 & 9 & $79.30 \pm 0.59$ & $82.94 \pm 0.37$ & $73.63 \pm 0.49$ & $72.13 \pm 0.59$ & $65.54 \pm 0.29$ \\
11 & 10 & $86.09 \pm 0.61$ & $89.15 \pm 0.64$ & $84.77 \pm 0.57$ & $76.54 \pm 0.69$ & $70.08 \pm 0.33$ \\
12 & 11 & $88.21 \pm 0.55$ & $92.26 \pm 0.46$ & $89.86 \pm 0.11$ & $80.48 \pm 0.75$ & $75.73 \pm 0.14$ \\
13 & 12 & $94.64 \pm 0.16$ & $96.18 \pm 0.38$ & $92.59 \pm 0.58$ & $86.96 \pm 0.79$ & $83.84 \pm 0.15$ \\
\hline & & & & & & \\
\hline
\end{tabular}

The data are presented as mean value \pm S.D. $(n=3)$

Table 9: Dissolution release profiles of Formulations (F11-F14).

\begin{tabular}{cccccc}
\hline \multirow{2}{*}{ S. } & Time(hrs) & \multicolumn{4}{c}{ Cumulative \% drug release*(\%) } \\
\cline { 3 - 6 } No & & F11 & F12 & F13 & F14 \\
\hline 1 & 0 & 0 & 0 & 0 & 0 \\
2 & 1 & $9.85 \pm 0.34$ & $7.23 \pm 0.51$ & $7.13 \pm 0.27$ & $7.65 \pm 0.41$ \\
3 & 2 & $13.06 \pm 0.27$ & $13.94 \pm 0.43$ & $16.71 \pm 0.81$ & $19.76 \pm 0.56$ \\
4 & 3 & $27.75 \pm 0.45$ & $29.06 \pm 0.25$ & $20.24 \pm 0.72$ & $29.82 \pm 0.39$ \\
5 & 4 & $30.71 \pm 0.14$ & $38.25 \pm 0.36$ & $23.16 \pm 0.63$ & $37.36 \pm 0.53$ \\
6 & 5 & $37.07 \pm 0.29$ & $49.68 \pm 0.58$ & $33.44 \pm 0.79$ & $45.48 \pm 0.27$ \\
7 & 6 & $51.52 \pm 0.16$ & $54.57 \pm 0.97$ & $38.16 \pm 0.51$ & $59.94 \pm 0.19$ \\
8 & 7 & $59.15 \pm 0.37$ & $69.77 \pm 0.77$ & $51.34 \pm 0.67$ & $67.82 \pm 0.22$ \\
9 & 8 & $63.65 \pm 0.25$ & $71.50 \pm 0.65$ & $62.36 \pm 0.44$ & $70.13 \pm 0.81$ \\
10 & 9 & $72.89 \pm 0.55$ & $82.60 \pm 0.63$ & $73.35 \pm 0.52$ & $74.36 \pm 0.42$ \\
11 & 10 & $78.33 \pm 0.64$ & $87.94 \pm 0.51$ & $79.81 \pm 0.49$ & $80.04 \pm 0.36$ \\
12 & 11 & $86.76 \pm 0.73$ & $90.67 \pm 0.72$ & $86.54 \pm 0.87$ & $83.29 \pm 0.47$ \\
13 & 12 & $94.16 \pm 0.27$ & $93.23 \pm 0.49$ & $91.55 \pm 0.23$ & $89.13 \pm 0.45$ \\
\hline
\end{tabular}

The data are presented as mean value \pm S.D. $(n=3)$

\section{CONCLUSION}

The Present Research work was to formulate and evaluate extended release Matrix tablets of Tenatoprazole sodium was prepared by wet granulation technique by using different polymers of Carbopol, HPMC and Eudragit grades. Formulations (F1-F18) fulfill the official limit for Physico Chemical parameters like weight Variation, hardness, friability and drug content uniformity. In-vitro dissolution studies showed that Tenatoprazole sodium tablets of in 1:1(Drug: Polymer) proportion, prepared by wet granulation method is the best to increase extended effect due to the polymer concentration. Formulation F5 (drug: Carbopol-974P-NF in ratio of 1:1.5) Shows extended drug release of $99.47 \pm 0.22 \%$ in 12 hours so it was selected as the best formulation among all the formulations.
Table 10: Dissolution release profiles of Formulations (F15-F18).

\begin{tabular}{cccccc}
\hline Time & Time(hrs) & \multicolumn{4}{c}{ Cumulative \% drug release*(\%) } \\
\cline { 2 - 6 } (hrs) & & F15 & F16 & F17 & F18 \\
\hline 0 & 0 & 0 & 0 & 0 & 0 \\
1 & 1 & $8.59 \pm 0.72$ & $9.21 \pm 0.49$ & $9.28 \pm 0.25$ & $9.22 \pm 0.51$ \\
2 & 2 & $17.56 \pm 0.67$ & $18.98 \pm 0.25$ & $13.40 \pm 0.41$ & $17.97 \pm 0.76$ \\
3 & 3 & $25.70 \pm 0.82$ & $29.85 \pm 0.45$ & $19.75 \pm 0.29$ & $28.22 \pm 0.25$ \\
4 & 4 & $39.05 \pm 0.46$ & $40.51 \pm 0.31$ & $26.05 \pm 0.36$ & $37.35 \pm 0.36$ \\
5 & 5 & $44.9 \pm 0.17$ & $52.28 \pm 0.42$ & $30.58 \pm 0.52$ & $44.10 \pm 0.39$ \\
6 & 6 & $58.54 \pm 0.27$ & $59.84 \pm 0.51$ & $40.04 \pm 0.55$ & $53.34 \pm 0.46$ \\
7 & 7 & $63.54 \pm 0.63$ & $68.87 \pm 0.35$ & $47.96 \pm 0.42$ & $62.23 \pm 0.62$ \\
8 & 8 & $65.47 \pm 0.33$ & $73.11 \pm 0.68$ & $58.45 \pm 0.37$ & $68.76 \pm 0.55$ \\
9 & 9 & $70.17 \pm 0.64$ & $81.29 \pm 0.59$ & $66.11 \pm 0.36$ & $73.38 \pm 0.38$ \\
10 & 10 & $74.36 \pm 0.67$ & $87.74 \pm 0.47$ & $72.74 \pm 0.59$ & $79.45 \pm 0.72$ \\
11 & 11 & $79.67 \pm 0.58$ & $91.66 \pm 0.35$ & $80.04 \pm 0.67$ & $84.56 \pm 0.89$ \\
12 & 12 & $85.75 \pm 0.69$ & $94.56 \pm 0.26$ & $84.74 \pm 0.83$ & $90.12 \pm 0.43$ \\
\hline
\end{tabular}

The data are presented as mean value \pm S.D. $(n=3)$

\section{ACKNOWLEDGEMENT}

The author would like to give sincere gratitude to the Progenerics Pharma Pvt. Ltd., Hyderabad, for providing all requirements for this Research work. I am also thankful to Brilliant Group of Institutions, R.R Dist, for providing valuable support in doing my Ph.D work.

\section{CONFLICT OF INTEREST}

The authors declared none.

\section{ABBREVIATIONS}

ER: Extended Release; PPI: Proton Pump Inhibitor; HPMC: Hydroxypropyl Methyl cellulose.

\section{REFERENCES}

1. Anroop N, Rachna G, Rachna K, Shery J, Mahesh A. Formulation and evaluation of enteric coated tablets of proton pump inhibitor. J of Basic and Clini Pharma. 2010; 1(4): 215-21.

2. Abul Bashar, Pulak Deb. Evaluation of Different Marketed Brands of Pantoprazole Sodium Tablets: A Comparative Study. ljppr. 2017; Vol. 9 (3): 134-40.

3. Bell NJ, Hunt RH. Role of gastric acid suppression in the treatment of gastro esophageal reflux disease. Gut.Bmj.1992; 33: 118-24.

4. Galmiche JP, Sacher-Huvelin S, Bruley des Varannes S. A comparative study of the early effects of tenatoprazole $40 \mathrm{mg}$ and esomeprazole $40 \mathrm{mg}$ on intragastric $\mathrm{pH}$ in healthy volunteers. Aliment Pharmacol Ther.2005; 21: 575-82.

5. Hunt $\mathrm{RH}$, Armstrong $\mathrm{D}$, James $\mathrm{C}$. Effect on intragastric $\mathrm{pH}$ of a $\mathrm{PPI}$ with a prolonged plasma half-life: comparison between tenatoprazole and esomeprazole on the duration of acid suppression in healthy male volunteers. Am J Gastroenterol. 2005; 100: 1949-56.

6. Dinesh Mohan S, Rama mohan gupta V, Manaswini Y. Impact of over coat Application on enteric coated drug pellets: Designed to protect from stomach environment. Int Res J Pharm. 2016; 7(10): 14-18.

7. Sourav Tribedi, Mahantesh, Sabitha JS, Rinku Mathappan, Prasanth VV. Formulation and evaluation of enteric coated tablets of pantoprazole. Int J Pharma and Chem Sci. 2013; 2(3): 1454- 61.

8. Vamshidhar Reddy D, Ambati Sambashiva Rao. Formulation and evaluation of extended release tablets of Tapentadol hydrochloride using hydrophilichydrophobic polymer combinations. J Pharm Res. 2014; 8(10): 1368-74.

9. Hashmat D, Shoaib MH, Mehmood ZA, Bushra R, Yousuf RI, Lakhani FM Development of Enteric Coated Flurbiprofen Tablets using Opadry /acryl-eze 
System - A Technical Note. AAPS Pharm Sci Tech. 2008;9(1):116-21.

10. Kranz H, Gutsche S. Evaluation of the drug release patterns and long term stability of aqueous and organic coated pellets by using blends of enteric and gastrointestinal insoluble polymers. Int J Pharm. 2009;380:112-9.
11. Dashevsky A, Kolter K, Bodmeier R. $\mathrm{P}^{H}$-independent release of a basic drug from pellets coated with the extended release polymer dispersion Kollicoat SR $30 \mathrm{D}$ and the enteric polymer dispersion Kollicoat MAE 30 DP. Eur J Pharm Biopharm. 2004;58:45-9.

Article History: Submission Date : 20-03-2020; Revised Date : 29-04-2020; Acceptance Date : 23-05-2020

Cite this article: Divya B, Sreekanth J, Satyavati D. Formulation and Evaluation of Extended Release Matrix Tablets of Tenatoprazole Sodium using Synthetic Polymers. J Young Pharm. 2020;12(2)Suppl:s39-s44. 